„Theorie sozialer Konflikte“, die Georg Simmels Arbeit über den Streit in eine moderne soziologische Sprache übersetzte und die konflikttheoretische Tradition in den Sozialwissenschaften mit begründete. Seine Dissertation sollte so erfolgreich werden, dass sie 1997 in die Liste der soziologischen Bestseller des 20. Jahrhunderts aufgenommen wurde. Auch wenn seine 18 Bücher und zahlreichen Artikel niemals den Erfolg seines Erstlings wiederholen sollten, so werden einige seiner Bücher unvergessen bleiben. 1963 gab er eine Studie über "Sociology through Literature“ heraus, die für die aufkeimende und mittlerweile wieder vergessene Literatursoziologie wichtig werden sollte, ähnlich einflussreich wie Hans Norbert Fügens Bemühungen in Deutschland. Es folgte in den 1970er Jahren seine Klassikerdiskussion „Masters of Sociological Thought“, die zusammen mit seinen „Men of Ideas" (erste Taschenbuchausgabe 1997) sicherlich zu den besten und lesbarsten Studien in diesem Feld gehören, in gleichem Atemzug zu nennen wie Raymond Arons „Hauptströmungen des soziologischen Denkens" oder Talcott Parsons' ,The Structure of Social Action". Kongenial ist auch das mit Bernard Rosenberg zusammengestellte Lehrbuch „Sociological Theory“ zu nennen. das 1982 in fünfter Auflage erschien und einem soziologischen Kanon gleich die wichtigsten soziologischen Texte versammelt. Mit Irving Howe verfasste er nicht nur eine Geschichte der kommunistischen Partei in Amerika, sondern machte auch Front gegen „The New Conservatives: A Critique from the Left". Seine institutionellen Erfahrungen an verschiedenen Universitäten des Landes, ja ihre Ambivalenz kommen in seinem kleinen, aber originellen Buch „Greedy Institutions“ zum Ausdruck. Wie eine Institution das Individuum aufzufressen vermag, kann jeder nachempfinden, der an einer deutschen Universität arbeitet. In den 1980er Jahren studierte er mit seinen Schülern Charles Kadushin und Walter Powell die Produktionsweise von Büchern: „Books: The Culture and Commerce of Publishing" - eine beeindruckende und informative kultursoziologische Arbeit. Ein Denk- und Mahnmal ganz eigener Art sollten seine „Refugee Scholars in America“ (1984) werden. Wer die Porttäts emigrierter Geistes- und Sozialwissenschaftler liest, wird Wut, Trauer und Aggression kaum unterdrücken können. Sie bilden das Mekka von Kultur, Geist und Bildung, das den kulturellen und wissenschaftlichen Aufschwung Amerikas nach dem Zweiten Weltkrieg mogglich machte. Bei der Lektüre dürfte auch dem letzten unverbesserlichen nostalgischen Hakenkreuzlet klar werden, dass Deutschland nur einen Todfeind wenn Lew Coser sich auf die Speerspitze der erfolgreichen Emigranten konzentriert, wird selbst hier, bei allem äußeren Erfolg, das Leiden an Deutschland, aber auch an der neuen fremden Heimat, deutlich. Die meisten von ihnen sind geblieben und nur wenige in das zerstörte Deutschland zurückgekehrt.

Auch Lewis A. Coser und seine Frau Rose Laub Coser sind nochmals nach Berlin zurückgekommen. Anlässlich der Georg-Simmel-Gastprofessur, die 1994 an der Humboldt-Universität inauguriert und zwischenzeitlich wegen Geldmangels wieder eingestellt wurde, erhielt Lew Coser die Ehrendoktorwürde. Nach sechzig Jahren der Vertreibung und dem 100-jährigen Jubiläum der Institutionalisierung der Soziologie an der Humboldt-Universität durch Georg Simmels ,Soziologische Übung" 1893/94 ein kleines Stück der Versöhnung - mit der deutschen Soziologie und dem von ihm hoch geschätzten Georg Simmel, mit seiner alten Heimatstadt und der neuen Hauptstadt des wieder vereinigten Deutschland, mit seiner Alma Mater, an der er doch nie studieren durfte. Lewis Cosers Kommentar als frisch gebackener Ehrendoktor: „Ich glaube, ab heute bin ich wieder ein Berliner."

Hans-Peter Müller

\section{Schaderpreis für Hartmut Häußermann und Walter Siebel}

Die Soziologen Hartmut Häußermann (HumboldtUniversität zu Berlin) und Walter Siebel (Carl von Ossietzky Universität Oldenburg) erhalten gemeinsam den Schaderpreis 2003. Mit dem Preis werden Gesellschaftswissenschaftler gewürdigt, die sich in besonderer Weise um den Dialog zwischen Sozialwissenschaften und Praxis verdient gemacht haben.

Seit 1978 haben die beiden Soziologen zu einer in der deutschen Wissenschaft ungewöhnlichen Publikationspartnerschaft zusammengefunden. Ihr Ertrag besteht in sechs gemeinsamen Büchern und nahezu fünfzig gemeinsamen Aufsätzen, von denen viele nicht vorrangig an die ,scientific community", sondern an die Kommunalpolitik, an Architekten und Planer, die Gewerkschaften, an die Kulturpolitik und die Öffentlichkeit gerichtet waren. Die Arbeiten beider Soziologen haben insgesamt wesentlich zur thematischen und konzeptuellen Ausrichtung der deutschen Stadtsoziologie beigetragen. 